\title{
Filter for Car Tracking Based on Acceleration and Steering Angle
}

Conference or Workshop Item

Accepted Version

Maybank, S.J., Worrall, A.D. and Sullivan, G.D. (1996) Filter for Car Tracking Based on Acceleration and Steering Angle. In: 7th British Machine Vision Conference, 9-12 Sept 1996, Edinburgh, pp10. Available at http://centaur.reading.ac.uk/2/

It is advisable to refer to the publisher's version if you intend to cite from the work. See Guidance on citing.

All outputs in CentAUR are protected by Intellectual Property Rights law, including copyright law. Copyright and IPR is retained by the creators or other copyright holders. Terms and conditions for use of this material are defined in the End User Agreement. 


\section{CentAUR}

Central Archive at the University of Reading

Reading's research outputs online 


\title{
Filter for Car Tracking Based on Acceleration and Steering Angle
}

\author{
S.J. Maybank, A.D. Worrall, G.D. Sullivan, \\ Department of Computer Science, \\ University of Reading, Whiteknights, \\ PO Box 225, Reading, Berkshire, RG6 6AY, UK \\ (S .J .Maybank, A.D.Worrall, G.D.Sullivan) Oreading .ac .uk
}

\begin{abstract}
The motion of a car is described using a stochastic model in which the driving processes are the steering angle and the tangential acceleration. The model incorporates exactly the kinematic constraint that the wheels do not slip sideways. Two filters based on this model have been implemented, namely the standard EKF, and a new filter (the CUF) in which the expectation and the covariance of the system state are propagated accurately. Experiments show that i) the CUF is better than the EKF at predicting future positions of the car; and ii) the filter outputs can be used to control the measurement process, leading to improved ability to recover from errors in predictive tracking.
\end{abstract}

\section{Introduction}

In systems for monitoring road traffic it is an advantage to have filters which can model accurately the motion of a car and predict its future positions. It becomes easier to track individual cars and to analyse their behaviour. Many current filters use over-simplified models based on general motion in the ground plane, without taking into account the specific nature of vehicle kinematics. An example can be found in[3]. These filters are easily distracted by bad measurements, and they tend to lose track of the car when it turns or when its speed changes sharply. This paper describes a new filter for tracking cars and predicting their future positions. The motion model is driven by the steering angle and the tangential acceleration. It incorporates exactly the constraint that the wheels of the car do not slip sideways during the motion.

An earlier version of the filter is described in [4]. There the driving processes are the orientation of the car in the ground plane and the tangential acceleration. The new model, developed below, gives a better description of the motion of the car at low velocities. In [4] the orientation of the car can change even when the tangential velocity is zero, i.e. the car can spin on the spot. The new model is more realistic, in that the orientation of the car cannot change at zero tangential velocity.

As in [4] the state of the car conditional on the measurements is approximated by a Gaussian random variable. The measurement process is assumed to be linear 


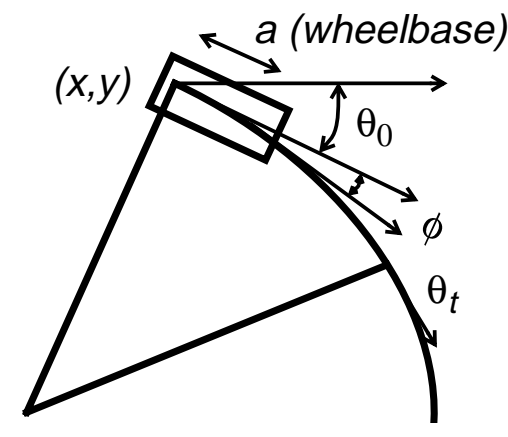

Figure 1 Vehicle kinematics: The instantaneous trajectory follows an arc whose radius is determined by the steering angle $(\phi)$ and the wheelbase $(a)$.

and subject to Gaussian noise. The measurements are incorporated using the standard Kalman update [5]. Also as in [4], we use a new mathematical technique for computing the evolution of the state expectation and covariance over time, the Covariance Update Filter (CUF). The CUF propagates the expectation and covariance of the state over a time interval $t$ with an error of magnitude $O\left(t^{3}\right)$. It offers greater accuracy than the conventional extended Kalman Filter (EKF).

This paper explores the accuracy of the CUF and the EKF using Monte Carlo simulations. It then shows how the filter output can be used to control the measurement process, thereby improving tracking performance.

\section{Model}

It is assumed that the car moves in the ground plane. The motion is modelled by a stochastic process $M=(X, Y, \Theta, V, \Phi, \dot{V})^{\top}$ in $\mathbb{R}^{6}$, where $(X, Y)^{\top}$ is the position of the car, $\Theta$ is the orientation, $V$ is the speed and $\Phi$ is the steering angle. The speed $V$ is positive for forward motion and negative for reverse motion. It is assumed that the initial state $M_{0}=\left(X_{0}, Y_{0}, \Theta_{0}, V_{0}, \Phi_{0}, \dot{V}_{0}\right)^{\top}$ is a Gaussian random variable. The motion geometry is illustrated in Figure 1.

Let $a$ be the wheelbase of the car. The driving processes are $\Phi, \dot{V}$. The remaining components of $M$ are obtained by integrating the following stochastic differential equations over time $t \geq 0$.

$$
\begin{aligned}
\left(d X_{t}, d Y_{t}\right)^{\top} & =V_{s}\left(\cos \left(\Theta_{s}\right), \sin \left(\Theta_{s}\right)\right)^{\top} d t \\
d \Theta_{t} & =a^{-1} V_{t} \Phi_{t} d t \\
d V_{t} & =\dot{V}_{t} d t
\end{aligned}
$$

The processes $\Phi, \dot{V}$ are Gaussian, and satisfy the equations

$$
d \Phi_{t}=-\alpha \Phi_{t} d t+\sigma d C_{t} \quad d \dot{V}_{t}=q d B_{t}
$$

where $(B, C)^{\top}$ is a Brownian motion independent of $M_{0}$, such that $\left(B_{0}, C_{0}\right)^{\top}=0$. The constants $\alpha, \sigma, q$ are chosen to suit particular car motions. 
The second equation of (1) is an approximation to the more accurate $d \Theta_{t}=$ $a^{-1} V_{t} \tan \left(\Phi_{t}\right) d t$, which arises from the constraint that the wheels of the car do not slip sideways [1]. The quantity $\kappa_{t}=V_{t}^{-1} d \Theta_{t} / d t$ is the curvature of the trajectory of the car. In a typical car $a \approx 2.5 \mathrm{~m}$ and $\kappa_{t} \geq 0.2 \mathrm{~m}^{-1}$, with the bound of $0.2 \mathrm{~m}^{-1}$ only reached at full lock. It follows that $\left|\tan \left(\Phi_{t}\right)\right| \leq 0.5$. The error in replacing $\tan (\Phi)$ by $\Phi$ is about $7 \%$ in the worst case, and it is usually much less.

\section{$3 \quad$ Expectations and Covariances}

In the CUF the estimates of the expectation and covariance of $M$ are propagated forward in time according to the equations given in this section.

The process $N \equiv(V, \Phi, \dot{V})^{\top}$ is the solution of a linear stochastic differential equation [2]. Let $A, \Sigma$ be the matrices defined by

$$
A=\left(\begin{array}{ccc}
0 & 0 & 1 \\
0 & -\alpha & 0 \\
0 & 0 & 0
\end{array}\right) \quad \Sigma=\left(\begin{array}{ccc}
0 & 0 & 0 \\
0 & \sigma & 0 \\
0 & 0 & q
\end{array}\right)
$$

The random variable $N_{t}$ is Gaussian with expectation $\exp (A t) E\left(N_{0}\right)$ and covariance

$$
\exp (A t)\left(\operatorname{Cov}\left(N_{0}, N_{0}\right)+\int_{0}^{t} \exp (-A s) \Sigma \Sigma^{\top} \exp \left(-A^{\top} s\right) d s\right) \exp \left(A^{\top} t\right) \quad(0 \leq t)
$$

The process $\Theta$ is non-Gaussian, however the expectation, $E(\Theta)$, and the covariances involving $\Theta$ and $V, \Phi, \dot{V}$ can be evaluated exactly. Let $\Psi$ be any random variable independent of $(B, C)^{\top}$, and let the functions $h_{i}, 0 \leq i$, and $c_{t}(\Psi), d_{s, t}$ be defined by

$$
\begin{aligned}
h_{i}(t) & =\int_{0}^{t} s^{i} \exp (-\alpha s) d s & & (0 \leq t) \\
c_{t}(\Psi) & =\operatorname{Cov}\left(\Psi, \Phi_{0} V_{0}\right) h_{0}(t)+\operatorname{Cov}\left(\Psi, \Phi_{0} \dot{V}_{0}\right) h_{1}(t) & & (0 \leq t) \\
d_{s, t} & =\operatorname{Cov}\left(\int_{0}^{s} V_{u} \Phi_{u} d u, \int_{0}^{t} V_{u} \Phi_{u} d u\right) & & (0 \leq s \leq t)
\end{aligned}
$$

Let $p, q:[0, \infty)^{2} \rightarrow \mathbb{R}$ be defined by

$$
\begin{array}{ll}
p_{s, t}=\operatorname{Cov}\left(q \int_{0}^{s} B_{u} d u, \int_{0}^{t} V_{u} \Phi_{u} d u\right) & (0 \leq s, t) \\
q_{s, t}=\operatorname{Cov}\left(\sigma \int_{0}^{s} \exp (\alpha u) d C_{u}, \int_{0}^{t} V_{u} \Phi_{u} d u\right) & (0 \leq s, t)
\end{array}
$$

It follows that for $0 \leq s, t$,

$$
\begin{aligned}
E\left(\Theta_{t}\right) & =E\left(\Theta_{0}\right)+a^{-1}\left(E\left(V_{0} \Phi_{0}\right) h_{0}(t)+E\left(\dot{V}_{0} \Phi_{0}\right) h_{1}(t)\right) \\
\operatorname{Cov}\left(\Theta_{s}, \Theta_{t}\right) & =\operatorname{Cov}\left(\Theta_{0}, \Theta_{0}\right)+a^{-1}\left(c_{s}\left(\Theta_{0}\right)+c_{t}\left(\Theta_{0}\right)\right)+a^{-2} d_{s, t} \\
\operatorname{Cov}\left(\Theta_{t}, V_{s}\right) & =\operatorname{Cov}\left(V_{0}, \Theta_{0}\right)+\operatorname{Cov}\left(\dot{V}_{0}, \Theta_{0}\right) s+\frac{1}{a}\left(c_{t}\left(V_{0}\right)+c_{t}\left(\dot{V}_{0}\right) s+p_{s, t}\right)
\end{aligned}
$$




$$
\begin{aligned}
\operatorname{Cov}\left(\Theta_{t}, \Phi_{s}\right)= & \exp (-\alpha s)\left(\operatorname{Cov}\left(\Phi_{0}, \Theta_{0}\right)+a^{-1} c_{t}\left(\Phi_{0}\right)+a^{-1} q_{s, t}\right) \\
\operatorname{Cov}\left(\Theta_{t}, \dot{V}_{s}\right)= & \operatorname{Cov}\left(\Theta_{0}, \dot{V}_{0}\right)+a^{-1} c_{t}\left(\dot{V}_{0}\right)+q^{2}(2 a)^{-1} E\left(\Phi_{0}\right) h_{2}(t) \quad(t \leq s) \\
\operatorname{Cov}\left(\Theta_{t}, \dot{V}_{s}\right)= & q^{2} a^{-1} E\left(\Phi_{0}\right) s\left(h_{1}(t)-h_{1}(s)-2^{-1} s\left(h_{0}(t)-h_{0}(s)\right)\right) \\
& +\operatorname{Cov}\left(\Theta_{s}, \dot{V}_{s}\right)+c_{t}\left(\dot{V}_{0}\right)-c_{s}\left(\dot{V}_{0}\right) \quad(s \leq t)
\end{aligned}
$$

The expressions (3) for the expectation and covariances involving $\Theta$ are complicated, however they can in practice be evaluated without difficulty using a computer algebra system such as Mathematica [7].

The expectations and covariances involving $X$ or $Y$ are more difficult to calculate than those involving $\Theta$. The calculations are reduced firstly by combining $X$, $Y$ to make a complex valued stochastic process $Z, Z=X+i Y$, where $i^{2}=-1$, and secondly by estimating expectations and covariance only to within an error of order $O\left(t^{3}\right)$.

It follows from (1) that

$$
Z_{t}=Z_{0}+\exp \left(i \Theta_{0}\right)\left(V_{0} t+\frac{1}{2} \dot{V}_{0} t^{2}\right)+\frac{1}{2} a^{-1} i \exp \left(i \Theta_{0}\right) V_{0}^{2} \Phi_{0} t^{2}+\ldots \quad(0 \leq t)
$$

Let $g$ be the function defined on Gaussian random variables $W$ by $g(W)=$ $\operatorname{Cov}\left(Z_{t}, W\right)$. The covariances of $Z_{t}$ with $\Theta_{t}, V_{t}, \Phi_{t}, \dot{V}_{t}, 0 \leq t$, are

$$
\begin{aligned}
\operatorname{Cov}\left(Z_{t}, \Theta_{t}\right) & =g\left(\Theta_{0}\right)+a^{-1}\left(\operatorname{Cov}\left(Z_{t}, V_{0} \Phi_{0}\right) h_{0}(t)+\operatorname{Cov}\left(Z_{t}, \dot{V}_{0} \Phi_{0}\right) h_{1}(t)\right) \\
\operatorname{Cov}\left(Z_{t}, V_{t}\right) & =g\left(V_{0}\right)+g\left(\dot{V}_{0}\right) t \\
\operatorname{Cov}\left(Z_{t}, \Phi_{t}\right) & =\exp (-\alpha t) g\left(\Phi_{0}\right) \\
\operatorname{Cov}\left(Z_{t}, \dot{V}_{t}\right) & =g\left(\dot{V}_{0}\right)
\end{aligned}
$$

\section{Calculation of Expected Values}

The expectations and covariances listed in $\S 3$ are often difficult to evaluate by hand because of the number of terms that arise when then are written out in terms of the expectations and covariances of the driving processes $\Phi$ and $\dot{V}$. Fortunately they can be evaluated efficiently using computer algebra. It suffices to consider only the evaluation of expectations of the form

$$
E\left(\prod_{i=1}^{n} A_{i}\right) \quad \text { or } \quad E\left(\exp \left(i A_{1}\right) \prod_{i=2}^{n} A_{i}\right)
$$

where the $A_{i}$ are Gaussian random variables.

Let $A=\left(A_{1}, \ldots, A_{n}\right)^{\top}, e=E(A), C=\operatorname{Cov}(A, A)$, and let $p=\left(p_{1}, \ldots, p_{n}\right)^{\top}$ be an $n$-dimensional vector of indeterminants $p_{i}, 1 \leq i \leq n$. Then it follows that

$$
E(\exp (i p . A))=\exp \left(-\frac{1}{2} p^{\top} C p+i p . e\right)
$$

Let $c_{n}$ be the coefficient of the product $p_{1} \ldots p_{n}$ in the Taylor series expansion of the right-hand side of (5). It follows from (5) that

$$
E\left(\prod_{i=1}^{n} A_{i}\right)=(-i)^{n} c_{n}
$$


To obtain the second expectation in (4), set $p_{1}=1$ on the right-hand side of (5). The resulting expression is expanded as a Taylor series in $p_{2}, \ldots, p_{n}$. Let $d_{n}$ be the coefficient of the product $p_{2} \ldots p_{n}$ in the expansion. Then it follows that

$$
E\left(\exp \left(i A_{1}\right) \prod_{i=2}^{n} A_{i}\right)=(-i)^{n-1} d_{n}
$$

\section{Experiments}

This section reports experiments to investigate the relative performance of the CUF and the EKF in estimating the behaviour of the kinematic model.

\subsection{Monte Carlo simulation}

Given a deterministic state $M_{0} \in \mathbb{R}^{6}$ at time zero, the kinematic model was run for a time $t$ to determine a realisation of the random variable $M_{t}$. On repeatedly running the model from the same starting point $M_{0}$, we obtained a distribution of states at time $t$. These provided an approximation to the distribution of $M_{t}$. Figure 2 (top two rows) illustrates results obtained from Monte Carlo experiments in which approximate realisations of $M=(X, Y, \Theta, V, \Phi, \dot{V})^{\top}$ were obtained with a timestep of $1 / 25$ sec. over a time interval of 1 sec. Each point is the projection of a final state of the model onto the $x, y$ plane. In all cases $\left(X_{0}, Y_{0}\right)^{\top}=(0,0)^{\top}$, $\Theta_{0}=\pi / 4$, and $V_{0}=4 \mathrm{~m} / \mathrm{sec}$; individual diagrams show different values of $\Phi_{0}$ and $\dot{V}_{0}$.

In Figure 2, top two rows, the best-fitting 2-D Gaussian is shown superimposed on the points (as a white line at the $1 \mathrm{sd}$. deviation contour). A $\chi^{2}$ test showed that the realisations of $M_{t}$ produced by the Monte Carlo simulation were not drawn from a Gaussian distribution. A typical set of 1000 runs with $\Phi_{0}=0$ and $\dot{V}_{0}=0$ yielded a $\chi^{2}$ value of 3166.2. Under the null hypothesis that the data are drawn from a Gaussian distribution, the random variable $G=\left(\chi^{2}-p\right) / \sqrt{2 p}$ is approximately Gaussian, with zero expectation and unit variance. The value realised by $G$ for the 1000 runs was 49.0, which is strong evidence for rejecting the null hypothesis.

Figure 2 (bottom two rows) reproduces the best-fitting Gaussian (magnified) as a grey ellipse occupying the area within $1 \mathrm{sd}$. deviation of the expected value, together with the estimates of the distribution obtained by the CUF and the EKF (black and white lines respectively). In all cases the EKF seriously underestimates the variance of the empirical data. The CUF provides a much more accurate estimate of variance.

The tendency of the EKF to underestimate covariance is "folklore" in computer vision, but we do not know of a reference. A consequence the underestimation is that the EKF pays insufficient attention to the measurements.

\subsection{Predictive Tracking}

The new kinematic model has been incorporated into a vehicle tracking system, using either the standard EKF [6] or the CUF. For each filter, the current estimate 


\section{British Machine Vision Conference}
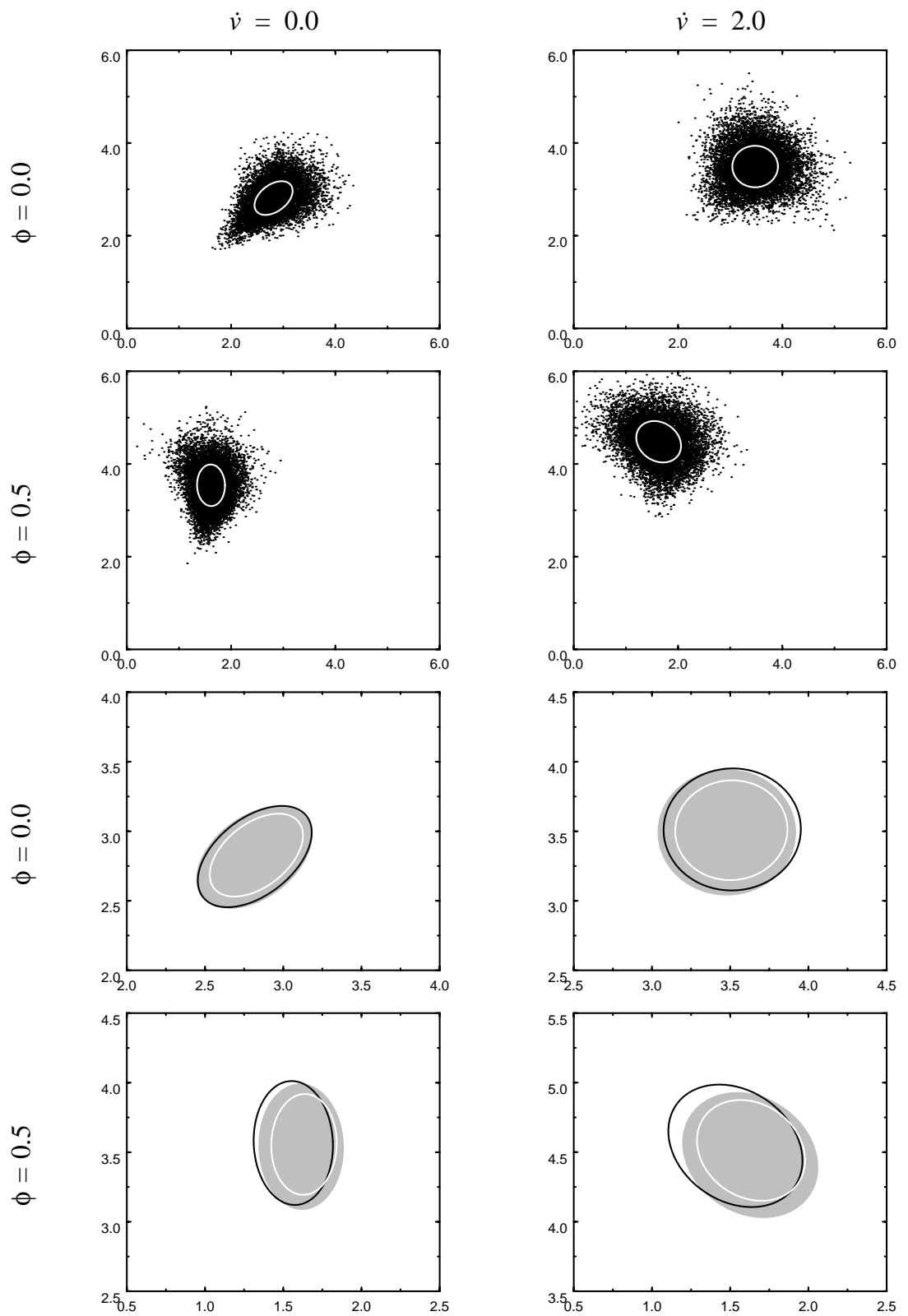

Figure 2 Top two rows: Monte Carlo trials of the kinematic model, projected onto the $(x, y)$ plane (black dots). Initial conditions $x=y=0, \theta=\pi / 4, v=4 m s^{-1}$ in all cases. Separate graphs for steering angle $\phi=(0,0.5)$ radians and acceleration $\dot{v}=(0.2 .0) \mathrm{ms}^{-2}$. Best-fitting ellipse at 1sd shown in white.

Bottom two rows: Same conditions, best fitting ellipses (grey) with covariance estimated by EKF (white) and the CUF (black). NB: change of scales. 


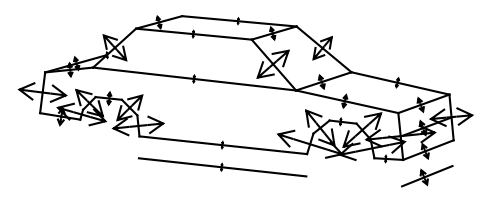

a

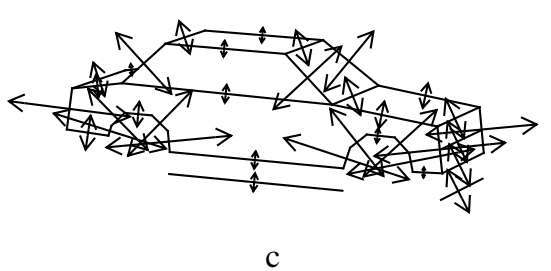

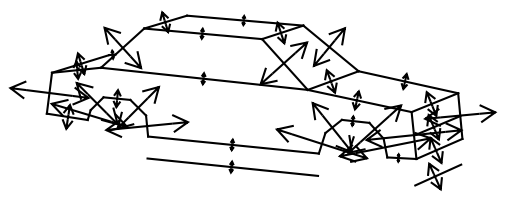

b

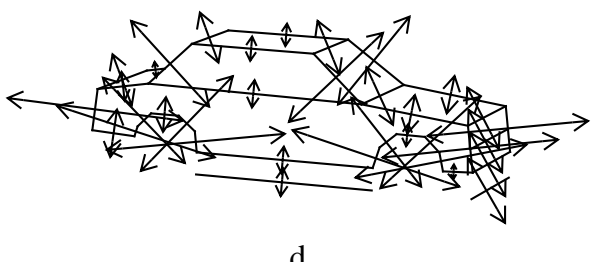

d

Figure 3 Search regions (perpendicular in the image to the projected model lines) determined by $+/-3$ sd of the estimated filter covariance for forward predictions of $(a, b, c, d)$ 0, 5, 10 and 15 fields (@50 Hz).

of the state vector was used to predict the expected pose of the vehicle in a new image. The prediction became the seed pose for model-based pose refinement, using the "active" method described in [8]. The recovered pose provided a measurement of $(x, y, \theta)^{\top}$ which was incorporated into the state estimate using the standard Kalman measurement update. For prediction over short intervals (20 to $60 \mu \mathrm{s}$ ) both filters worked extremely well, even in sequences in which the vehicle underwent sudden changes of behaviour, for example during a three point turn.

\subsection{Control of the Measurement Process}

The pose recovery technique in $[4,8]$ performs a standard search for high image derivatives close to the lines of the projected model. However, an accurate estimate of the covariance of the pose provides a means to control the search, taking account of the current uncertainty.

Let $p_{0}, C_{0}$ be the expectation and covariance of the pose $p$, let $l(p)$ be the projection of a model line and let $n_{0}$ be a unit vector normal to $l_{0} \equiv l\left(p_{0}\right)$. The neighbourhood of each line $l_{0}$ was searched for points at which the image derivative in the direction $n_{0}$ is large. The method was to choose points $r_{0}$ uniformly spaced on $l_{0}$ and to search along each normal $r_{0}+\lambda n_{0}$. The covariance $C_{0}$ was used to fix the range $-\lambda_{m} \leq \lambda \leq \lambda_{m}$ for the search; $\lambda_{m}$ was the largest value of $\lambda$ such that $r_{0}+\lambda n_{0}$ is on $l(p)$, and such that $\left(p-p_{0}\right)^{\top} C_{0}^{-1}\left(p-p_{0}\right)=9$. A linear approximation

$$
l(p)=l_{0}+\left.\frac{\partial l}{\partial p}\right|_{p_{0}}\left(p-p_{0}\right)+O\left(\left\|p-p_{0}\right\|^{2}\right)
$$

was made and $\lambda_{m}$ was estimated by minimising a quadratic in $\lambda$.

Typical consequences are shown in Figure 3, where the search regions are shown for forward predictions of $0,5,10$ and 15 video fields. The 0 case indicates search areas based only on the current uncertainty of the filter state; 5, 10 and 15 show 
Extended Kalman Filter
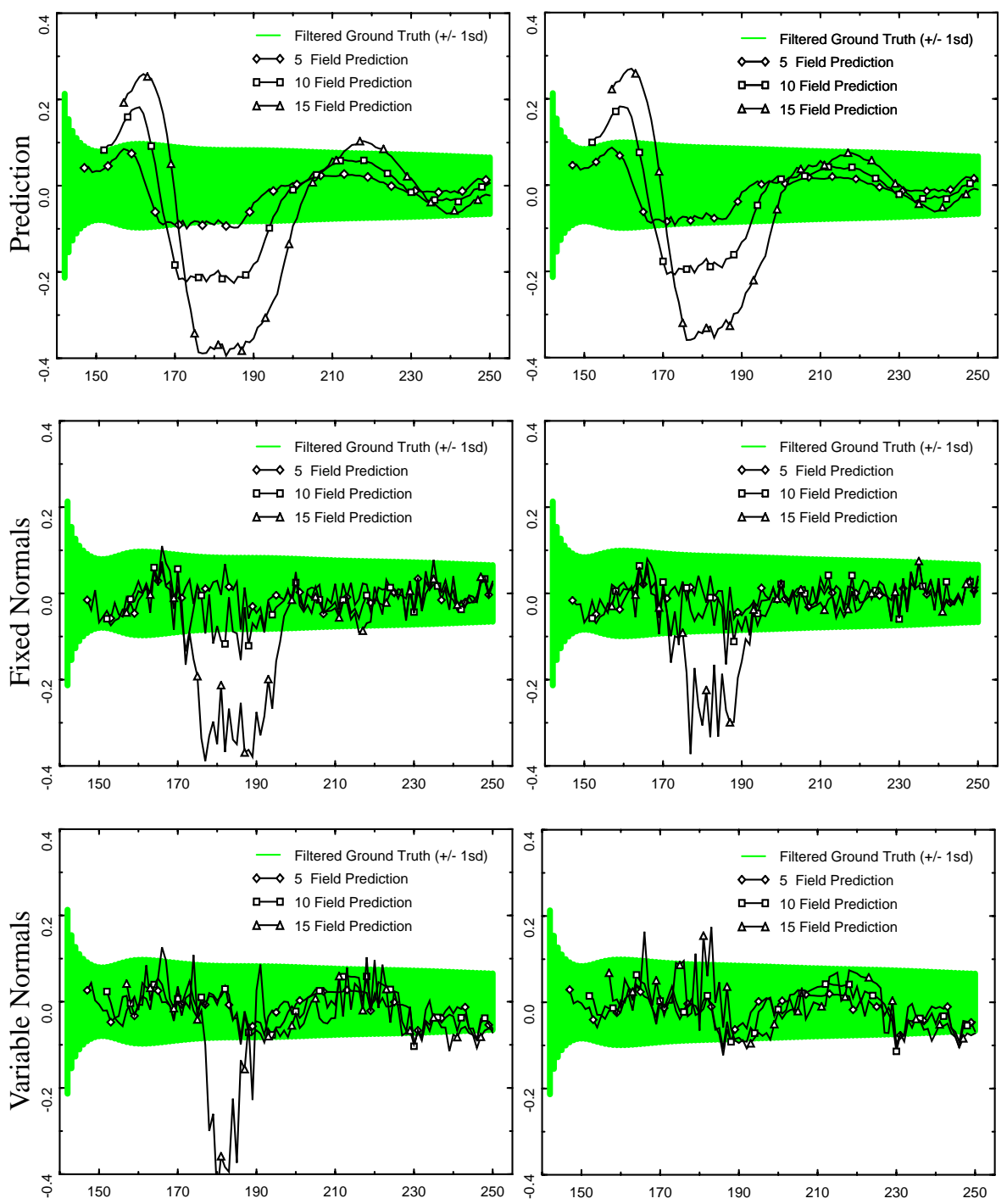

Figure 4 Performance of the kinematic model using the EKF estimator (left); and the CUF estimator (right). "Ground truth" is given by ordinate $=0.0$ (scaled in metres), and +/- 1 sd. of the estimated y-parameter is shown shaded.

Top: $\quad$ Forward predictions for 5, 10, and 15 fields (@50 Hz).

Middle: Recovered poses using fixed length normals (+/-4 pixels).

Bottom: Recovered poses using search regions controlled by the current estimate of filter covariance. 
how the search areas grew rapidly as the noise sources in the filter take effect. Note that many lines (e.g. the roof of the car) were relatively unaffected by legitimate changes of pose allowed by the filter, whereas others (e.g. near vertical lines in this pose) were strongly affected.

\subsection{Comparisons of Performance}

A short four way experimental assessment of the techniques has been carried out using a video sequence of a car coming into a corner and slowing down. The $25 \mathrm{~Hz}$ video sequence was deinterlaced by creating full sized fields in which the missing lines were replaced by the averages of their neighbours. Images were therefor available at $50 \mathrm{~Hz}$.

The four cases examined the EFK and the CUF, both using either a fixed search distance of \pm 4 pixels (previously regarded as standard) or by controlling the normal search as described in $§ 5.3$. In each case the filter was conditioned by "ground truth" data, determined in advance, using images spaced one field apart (i.e. at $50 \mathrm{~Hz}$ ). At any given time, each filter was run forward to predict 5,10 and 15 fields ahead (top row of Figure 4), and then pose refinement was carried out. Results are illustrated in Figure 4 (bottom two rows) for one of the three pose parameters, namely the $y$ coordinate in the ground plane. The results for $x$ and $\theta$ are similar. Each graph shows, for a given video field, the error in the recovered pose, when the search is begun from the pose predicted by the filter. The prediction is based on ground truth data up to 5, 10 and 15 fields previously, as filtered by the appropriate filter. Errors are shown with respect to the filtered ground truth ( 0.0 on the ordinate) and the $\pm 1 \mathrm{sd}$. deviation of the currently filtered parameter (here $y$ ) is shown shaded. Note that the ground truth data was obtained using fixed normals so that the results are likely to be biased against variable normals.

The results show three main things.

i) The prediction error of the CUF is better than that of the EKF; note that both filters initially overshoot, but the CUF recovers more rapidly (Figure 4, top row).

ii) Improved predictions lead to better pose recovery using the CUF; note the pose recovery fails for a forward prediction of 15 fields, but the failure is less for the CUF (Figure 3, bottom two rows, right) than for the EKF (Figure 3 , bottom two rows, left).

iii) The variable normal search technique outperforms fixed normal search at long prediction times; note that there are fewer failures using variable normals (Figure 3, bottom row) for the CUF than for the EKF.

\section{Conclusion}

The main conclusion of this paper is that the kinematic model for car motion described in $\S 2$ is detailed and accurate enough to be the basis of a predictive filter to track vehicles undergoing complex motion, for example a three point turn.

In addition, we have shown that predictions of expectation and covariance can be made accurately to $O\left(t^{3}\right)$, using a technique we have called the Covariance 
Update Filter (CUF). By means of Monte Carlo simulations we have shown that the CUF is better able to describe the behaviour of the kinematic model than the EKF. Finally, a technique for using the current filter state to control the measurement process is outlined and shown to improve the performance still more.

\section{References}

1. H. Heisler 1989 Advanced Vehicle Technology. Hodder and Stoughton: London, Melbourne, Auckland.

2. P.E. Kloeden and E. Platen 1992 Numerical Solution of Stochastic Differential Equations. Applications of Mathematics 23. Springer-Verlag: Berlin.

3. H. Kollnig and H.-H. Nagel 1996 Matching object models to segments from an optical flow field. In B. Buxton and R. Cipolla (eds.) Computer VisionECCV'96, Lecture Notes in Computer Science 1065, 388-399. Springer-Verlag: Berlin, Heidelberg, New York.

4. S.J. Maybank, A.D. Worrall and G.D. Sullivan 1996 A filter for visual tracking based on a stochastic model for driver behaviour. In B. Buxton and R. Cipolla (eds.) Computer Vision-ECCV'96, Lecture Notes in Computer Science 1065, 540549. Springer-Verlag: Berlin, Heidelberg, New York.

5. P.S. Maybeck 1979 Stochastic Models, Estimation and Control - Volume 1. Mathematics in Science and Engineering Series 141-1. San Diego, CA, USA: Academic Press.

6. P.S. Maybeck 1982 Stochastic Models, Estimation and Control - Volume 3. Mathematics in Science and Engineering Series 141-3. London, UK: Academic Press.

7. S. Wolfram 1991 Mathematica: a system for doing mathematics by computer. Second edition. Addison Wesley: Redwood City, CA.

8. A.D. Worrall, J.M. Ferryman, G.D. Sullivan and K.D. Baker 1995 Pose and structure estimation using active models. In (ed. D. Pycock) British Machine Vision Conference 1995 1, 137-146. BMVA. 